- compared to controls. This measure of efficacy is recommended for assessing a partially effective vaccine ${ }^{4}$. But the public expects vaccine efficacy to describe protection over a period of time, argues Judith Epstein, a captain and paediatrician at the US Military Malaria Vaccine Program in Silver Spring, Maryland. Recalculating the trial data shows that RTS,S protected just $35-36 \%$ after 12 months, she says, adding that the paper should have presented both numbers. The study also showed no detectable impact on mortality, and it is too early to tell whether RTS,S actually protects against malaria, or merely delays infection.

The paper did report that RTS,S reduced severe malaria by $47 \%$ in the older group. But combining that result with available data from the younger age group cut that number to $34.8 \%$ - meaning that for the youngest children, the benefit must be even smaller. "The real question mark is the $34.8 \%$ efficacy in severe disease," says Blaise Genton of the Swiss Tropical and Public Health Institute in Basel, and a member of the WHO technical advisory group for RTS,S. The results suggest that the vaccine might fall short of expectations, laid out in 2006 by a WHO-led consortium ${ }^{5}$, that it should have a "protective efficacy of more than $50 \%$ against severe disease and death and lasts longer than one year". "If it doesn't reduce deaths, and has only a modest effect on severe malaria, these are going to be big questions for decision-makers at WHO, GSK and the Gates Foundation," says Hoffman.

Another worrying finding is that the frequency of serious adverse events, such as convulsions and meningitis, was significantly higher in the vaccinated group, although the data are too preliminary to draw firm conclusions. "The severe disease findings are a concern," says Genton.

But Hoffman, like many researchers contacted by Nature, says that RTS,S still marks a significant achievement. It is the first vaccine against a parasite, Plasmodium falciparum, to consistently show a significant protective effect in large-scale trials. The phase III trial of RTS,S resulted in groundbreaking cooperation with African scientists, who led the 11 trials in 7 countries, says Hoffman. "I think that those teams deserve an incredible amount of recognition and congratulation." 1. The RTS,S Clinical Trials Partnership New
Engl. J. Med. http://dx.doi.org/10.1056/ NEJMoa1102287 (2011)

2. Farlow, A. In The Science, Economics, and Politics of Malaria Vaccine Policy (2006). Chapter available at http://go nature com/8oxle3

3. White, N. J. New Engl. J. Med. http://dx.doi. org/10.1056/NEJMe1111777 (2011).

4. Moorthy, V. S., Reed, Z. \& Smith, P. G. Vaccine 27, 624-628 (2009)

5. World Health Organization et al. Malaria Vaccine Technology Roadmap (2006). Available at http://go.nature.com/i1jdf7

\title{
Fetal gene screening comes to market
}

\author{
Non-invasive procedure could make prenatal testing easier, \\ but it comes with ethical problems.
}

\section{BY ERIKA CHECK HAYDEN}

$\mathrm{U}$ ntil last week, scrutinizing a fetus's DNA for indications of genetic abnormalities meant tapping into the mother's womb with a needle. Now there's a test that can do it using a small sample of the mother's blood. MaterniT21, a Down's syndrome test that Sequenom of San Diego, California, launched in major centres across the United States on 17 October, is the first of several such tests expected on the market in the next year. It signals the arrival of a long-anticipated era of non-invasive prenatal genetic screening, with its attendant benefits and ethical complications (see Nature 469, 289-291; 2011).

With the technology in place to sequence the fetal DNA carried in a pregnant woman's bloodstream, geneticists predict the list of conditions that can be detected by non-invasive means will grow rapidly. Another company, Gene Security Network of Redwood City, California, says its forthcoming test will also check for other genetic abnormalities, and Sequenom is studying the feasibility of expanding its test.

"There's every reason to think that in the future you'll be able to extract an enormous amount of information from that sequencing data," says Peter Benn, director of the Diagnostic Human Genetics Laboratories at the University of Connecticut Health Center in Farmington.

Sequenom's test sequences 36-base-pair fragments of DNA to identify sections from chromosome 21. Normally, the chromosome contributes $1.35 \%$ of the total maternal and fetal DNA in the mother's blood. An overabundance of this material indicates the genetic abnormality that marks Down's syndrome.

Sequenom is marketing its test as an add-on to current screening methods, which estimate the chance that a woman is carrying a fetus with Down's syndrome from ultrasound results and protein markers in the blood. Such nongenetic screening can detect $90-95 \%$ of Down's syndrome cases, but falsely indicates that up to $5 \%$ of women are carrying a baby affected by the condition. Sequenom's test could be taken after a positive screening result to help a woman decide whether to undergo amniocentesis, a test that extracts amniotic fluid with a needle and carries a small risk of miscarriage. A study published this month, and paid for by

Sequenom, found that the company's test has a false positive rate of $0.2 \%$ (G. E. Palomaki et al. Genet. Med. http://dx.doi.org/10.1097/ GIM.0b013e3182368a0e; 2011).

It could spare some women from having amniocentesis after a false-positive screening result. But Benn says that the test will also pose difficulties. For instance, because it would take 8-10 days to get the results of Sequenom's test, if a woman did still opt for amniocentesis, and the result confirms that the baby has Down's syndrome, there would be little time left to decide whether to terminate the pregnancy. And some women who test positive on MaterniT21 will probably choose to terminate pregnancies immediately rather than have amniocentesis.

"Inserting this new test in the way that Sequenom is proposing is very difficult, from the patient perspective, and difficult for physicians and counsellors to manage," Benn says.

Ethicists also caution that using such easy

"In the future you'll be able to extract an enormous amount of information from that sequencing data." whether to carry an 'unhealthy' fetus to term.

"The idea that couples have choices about whether to continue their pregnancies may become strained because parents may be seen as irresponsible for allowing 'defective' pregnancies to go to term," says Mildred Cho, an ethicist at Stanford University in Palo Alto, California. Other ethicists worry that fears of eugenics will be raised if testing can be done for less-serious conditions.

Sequenom is solely focused on developing tests for conditions that are already part of prenatal screening programmes, says Mathias Ehrich, the company's senior director for research and development diagnostics. "We do not want to invent new applications. Our focus is on making existing clinical applications safer," he says. "I don't think that we are in a position to say that we should determine what hair colour the baby has." - 